\title{
CLINICAL CASE REPORT
}

Supporting holistic care for patients with tuberculosis in a remote Indigenous community: a case report

\section{AUTHORS}

Andrea Miller ${ }^{1}$ FRACGP, FARGP (Adv Skill Adult Internal Medicine), Senior Medical Officer

Alice Cairns ${ }^{2}$ PhD, HOT North Research Fellow *

Annah Richardson ${ }^{3}$ Master of Nursing (Rural and Remote), Clinical Nurse

Jill Lawrence ${ }^{4}$ Aboriginal and Torres Strait Islander Indigenous Health Worker

\section{CORRESPONDENCE}

*Dr Alice Cairns alice.cairns@jcu.edu.au

\section{AFFILIATIONS}

${ }^{1}$ Weipa Integrated Health Services, PO Box 341, Weipa, Queensland 4874, Australia

${ }^{2}$ Centre for Rural and Remote Health, James Cook University, Weipa Hospital PO Box 341, Weipa, Queensland 4874, Australia

${ }^{3}$ Weipa Integrated Health Service, Lot 497 John Evans Drive, Weipa, Queensland 4874, Australia

${ }^{4}$ Napranum Primary Health Care Clinic, Munding Road, Napranum, Queensland 4874, Australia

\section{PUBLISHED}

26 February 2020 Volume 20 Issue 1

HISTORY

RECEIVED: 11 July 2019

REVISED: 28 December 2019

ACCEPTED: 28 January 2020

\section{CITATION}

Miller A, Cairns A, Richardson A, Lawrence J. Supporting holistic care for patients with tuberculosis in a remote Indigenous community: a case report. Rural and Remote Health 2020; 20: 5552. https://doi.org/10.22605/RRH5552

This work is licensed under a Creative Commons Attribution 4.0 International Licence

\section{ABSTRACT:}

Context: Tuberculosis (TB) is a serious infectious disease with high rates of morbidity and mortality if left untreated. In Australia, TB has been virtually eradicated in non-Indigenous Australian-born populations but in remote Aboriginal and/or Torres Strait Islander communities TB presents a rare but significant public health issue.
Remote health services are most likely to encounter patients with suspected and confirmed TB diagnosis but may be unprepared for supporting someone with this disease and the complexities of balancing public health risk with patient autonomy.

Issue: This case study will outline the process for diagnosis and 
treatment of a TB patient in a remote Cape York community. This case involved significant delay in diagnosis and required several strategies to achieve successful disease eradication. The process of treatment, however, had a significant effect on the patient's physical health, and social and emotional wellbeing.

Lessons learned: This case highlights the importance of early collaboration between medical, nursing, Indigenous health worker and allied health services and the importance of technology such Keywords:

management, Queensland, tuberculosis.

as electronic information records to support opportunistic access to diagnostic services and treatment. The enactment of the TB protocol should include discussions about the consequences of any restrictions of movement, employment or social/community roles. Identifying alternative opportunities to engage in meaningful roles may reduce the impact the disease has on a patient's quality of life.

\section{FULL ARTICLE:}

\section{Context}

Mycobacterium tuberculosis is one of the most ancient bacteria to infect humans, and those without treatment of active infection have a 5 -year survival of less than $50 \%{ }^{\mathbf{1}}$. Tuberculosis (TB) remains one of the primary causes of mortality in developing countries ${ }^{2}$. TB diagnosis and treatment are unfamiliar to most urban Australian health practitioners; this disease, virtually eradicated from the Australian medical lexicon by the $1980 \mathrm{~s}^{3}$. Of contemporary cases in Australia, $90 \%$ occur in those born overseas (migrants) ${ }^{4}$. The remaining $10 \%$ of cases occur predominantly in Indigenous populations, at a rate six times that of non-Indigenous Australians ${ }^{4}$. Indigenous people living in Cape York and on the Torres Strait Islands have historically higher rates of TB than people in other parts of Australia 4 . Proximity to Papua New Guinea, which has a TB prevalence among one of the highest in the world, and the social and biological factors increasing risk of transmission, continues to put these communities at risk of this communicable disease $\mathbf{5}^{\mathbf{2}}$

Public health guidelines recommend anti-TB treatment commence within 3 days of diagnosis of active disease to mitigate the risk of transmission, with medication delivered as directly observed therapy in daily or three times weekly dosing ${ }^{6-8}$. The treatment cure rate for Indigenous people in Australia in recent years has been approximately $10 \%$ less than for non-Indigenous groups 4 . One primary contributor to treatment failure is non-adherence to medication, increasing the potential for the evolution of multidrug resistant $\mathrm{TB}$, an increasingly global concern. A study published in 2015 reported 13\% of cultured TB in Australia was drug resistant to first line anti-TB agents ${ }^{4}$. Challenges to medication adherence are multifactorial and include structural factors such as organisation of treatment and care for patients; and individual/patient factors such as patient interpretations of illness and wellness, knowledge, attitudes and beliefs about treatment, personal characteristics and adherence behaviour, influence of side effects on treatment adherence, and family, community and household influences ${ }^{9}$.

There are considerable challenges for health professionals who treat patients with TB, as they attempt to balance public health concerns and the maintenance of patient autonomy and quality of life. The remote environment and the cultural complexities of providing health care to Indigenous Australians compound these challenges. Indigenous people demonstrate lower TB cure rates than other populations, with increased vulnerability of remote Indigenous communities to disease outbreaks. There is a considerable body of literature exploring these issues in developing countries but little documentation of these complexities in the Australian remote Indigenous context.

This case report will explore the challenges of diagnosis and coordination of treatment of TB in a very remote Indigenous community and the impact this process had on the physical, social and emotional wellbeing of an individual. The patient has provided written consent for the publication of this case report.

\section{Issue}

The index case is a middle-aged Aboriginal woman residing in a remote Cape York Indigenous community. She initially presented to the local primary healthcare centre (PHCC) with cough and fever, at which time a local outpatient chest radiograph demonstrated a novel apical lung lesion. Multiple serial radiographs were obtained over a few months, with non-resolution of the lesion, with a $\mathrm{CT}$ radiograph recommended for definitive diagnosis, a service that requires transfer to the closest tertiary hospital, more than $800 \mathrm{~km}$ away. The patient did not attend for this scan and was lost to follow-up. The patient re-presented to the PHCC 3 years later and a CT chest radiograph, with findings suggestive of TB, was completed. Sputum sample smears confirmed Mycobacterium tuberculosis 3 months post-CT. Although at this stage she was asymptomatic, these results confirmed what appeared to be an autochthonous case of active pulmonary TB from an unknown source.

As per guidelines and under the remote supervision of the stateled TB control unit, the patient was admitted to the local remote hospital to commence the first 2 weeks of drug treatment. Attempts at isolating her in hospital for induction treatment proved unsuccessful as the patient self-discharged. The patient continued to engage with the health staff in the community although her adherence to medical treatment and appointments was inconsistent. To reduce the public health risk, the TB unit advised the patient to cease her welfare/government supported employment at the local childcare centre. The loss of her work role appeared to precipitate an increase in alcohol and cannabis use, resulting in chaotic social interactions and loss of routine. This 
complicated the medical management of this patient, with the health service eventually using a modified directly observed therapy (DOT) approach to optimise medication adherence.

\section{Treatment strategies}

Following the initial failed hospital admission, DOT was trialled in the community. Initially, a clinical nurse consultant, with support from an Indigenous Health Worker (IHW), delivered medication Monday to Friday with weekend medication left with the patient on a Friday. This resulted in suboptimal adherence as locating the patient was problematic. The period directly after diagnosis and subsequent withdrawal from work was the most chaotic for the patient. The patient's loss of a consistent daily structure that would ordinarily support treatment adherence significantly affected the delivery of treatment in the first few months.

Alternative treatment regimens were trialled, including three times weekly treatment and provision of take-home medications for weekends; however, there remained uncertainty with adherence when self-administering. Finally, in consultation with the patient, the use of a pre-packed medication system (Webster-pak) was employed. Initially the registered nurse and an IHW observed selfadministration of medications from the Webster-pak daily. As the use of the Webster-pak did not require a registered nurse to administer medication, the case management of the patient care was able to be transferred to the IHW, who was able to build a relationship with the patient to facilitate more reliable treatment adherence. Visits were short (approximately $5 \mathrm{~min}$ ) but daily. Through building a medication routine acceptable to the patient, the IHW could gradually reduce the level of support provided to the patient to ensure medication adherence. This short-term intensive support to develop a medication routine had positive unintended consequences such as improving the consistency of the patient's daily routine, reducing alcohol and improving her nutritional intake. The patient also re-engaged with her employment provider. She ceased medication after 14 months of therapy and thus far is considered cured of TB, for ongoing surveillance.

\section{Lessons learned}

This case report describes the diagnosis of TB in an Aboriginal person residing in a remote community in Cape York and the treatment strategies that resulted in successful disease eradication. Although the desired outcome of cure was achieved, limited access to timely and appropriate health services and the lack of engagement with the patient outside of their immediate medical needs resulted in delayed diagnosis, extended treatment requirements and significant disruption to the patient's work and social roles.

Two interrelated categories can be used as a framework for exploring the challenges faced by the patient and health service in treating this case: the health service and environmental factors; and individual, personal and lifestyle factors.

\section{Health service and environmental factors}

Several health service factors were identified through this case study as impacting on the provision of best practice for the patient. These included staff unfamiliar with diagnosis and treatment of TB, poor access to diagnostic tools such as CT scan, paper-based records restricting timely communication between local hospital and primary health care staff, and a lack of multidisciplinary involvement to reduce the burden of the disease on the patient's social and occupational roles.

Both patient delay in seeking health care and health systems delay contribute to delayed diagnosis and treatment, potentially increasing morbidity and public health risk ${ }^{\mathbf{1 0 - 1 2}}$, as in this case. Previous research has reported Indigenous Australians have been less likely to experience a delay in diagnosis than other groups ${ }^{\mathbf{1 3}}$. When this patient initially presented with cough and fever, and radiographical evidence of an apical lung lesion, the index of suspicion for TB should have been higher, with unfamiliarity and the relative rarity of this illness in Cape York possibly contributing to this delayed diagnosis. Access to CT imaging for all residents in Torres Strait and Cape York involve air travel to Cairns and at least one overnight stay. This can be a significant burden for patients who are responsible for caring for others (children, elders) or have other family or community responsibilities, and navigating the complexities of tertiary level services and location has previously been demonstrated to present a barrier to accessing services for remote Indigenous residents ${ }^{\mathbf{1 4}}$. Initiation of treatment was also compromised by distance, as medications took time to arrive from Cairns and did not arrive prior to the patient's self-discharge, resulting in further delays to continuation of treatment and an associated increase in public health risk.

In Australia, following the diagnosis of active TB, treatment is recommended to commence within 72 hours ${ }^{\mathbf{6}}$, with a 2 -week hospitalisation recommended at the initiation of treatment. These protocols exist out of context to this patient's remote community setting and hospital service and in this case the execution of the TB protocol was not well considered from an organisational perspective. Due to unfamiliarity, health service staff experienced reduced comfort levels with respect to managing this patient. This resulted in staff focusing on the biomedical and risk management of this infectious disease without considering how to best support someone, who felt relatively well, to stay in hospital for 2 weeks. Two attempts at hospitalisation resulted in self-discharge despite the patient's willingness to treat the TB and agreeing to the admissions. In hindsight, this was unsurprising as there was no discussion with the patient or health staff about how the patient would spend their time in hospital. There was no referral to social work for input and at the time the health service did not have an occupational therapy service that could support this patient to maintain occupational roles during the treatment process.

The combination of DOT and a case management model better served the needs of this patient and ensured her care was maintained within the complicated context of this remote health service. Strategies used by the case manager involved raising issues regarding patient engagement at the daily primary care staff meetings and weekly multidisciplinary team meetings. The success of case management models in the treatment of TB, especially 
when used in conjunction with DOT, has been reported within the literature ${ }^{15}$ and this model certainly reduced fragmentation of communication in this case (a problem accentuated by separate paper-based records between the hospital and PHCC). A lack of shared electronic medical records and electronic patient recall system meant that there was a heavy reliance on verbal and email communications, which were not always filed in a timely manner or sent to all relevant parties involved in patient care. This not only resulted in disjointed provision of service and missed opportunities for patient review, but also occasionally put staff at risk who were not aware that this patient, who presented sporadically to the emergency department, required airborne precautions for TB. It has been suggested that a common electronic platform of communication can improve management of TB, especially with respect to the complicated drug regimens in multi-drug resistant $\mathrm{TB}^{\mathbf{1 6}}$, and this certainly would have been helpful in this case.

\section{Individual, personal and lifestyle factors}

Individual and cultural factors affect the adherence to treatment in $\mathrm{TB}^{9}$. In this case, the patient was agreeable to treatment, attended medical appointments and was open to discussions about her illness and the treatment regimen. Regardless of this cooperation, it became quickly obvious that competing needs of cultural and personal priorities and the adherence to the intensity of the treatment were going to place the patient at risk of treatment failure and development of multi-drug resistant TB. The health priorities for many patients with chronic conditions are not that of disease control and eradication but the maintenance of daily social activities ${ }^{\mathbf{1 7}}$. Stigma from the community can limit willingness to engage in treatment ${ }^{\mathbf{1 8}}$, and a 'shame' factor was identified in this case as a significant barrier to daily DOT, with the patient embarrassed by being singled out by the health service in front of her family. As observed in this case with the transition of case management to the Indigenous health worker, the provision of a culturally competent health service in-conjunction with medication adherence strategies (Webster-pak, simplified dosing), can improve treatment outcomes in Indigenous patients ${ }^{\mathbf{1 7}}$.

Due to the perceived public health risk in this case, the patient was advised to withdraw from her role as a childcare worker, resulting in disrupted interaction with others and a degree of social isolation, with no redress for this offered by the health service. In patients with TB, as with many diseases, unemployment and low socioeconomic status are associated with a lower baseline healthrelated quality of life, and the psychosocial burden can sometimes be greater than the physical burden ${ }^{\mathbf{1 9}}$. The advice to cease employment was provided without the support of a social worker or occupational therapist. If the public health risk requires patients to restrict work or other social roles, efforts should be made to ensure this is implemented in consultation with relevant stakeholders and to enlist support of a multidisciplinary team to mitigate the financial and psychosocial losses to the patient. In some cases, it may be possible to negotiate alternative employment or social roles, which may have been helpful in this case.

\section{Conclusion}

This case illustrated the challenges of diagnosis and management of TB in a remote Indigenous community in Far North Queensland, including delays in diagnosis related to the remote environment, multiple paper-based record systems, and a focus on biomedical needs and individual patient factors. In very remote northern Australia, a model to support timely diagnosis with an increased threshold for suspicion of TB would be appropriate. Diagnostic support, ideally with local access to CT services, is equally as important as an effective treatment model with respect to reducing public health risk and patient morbidity. A single electronic medical record could have effectively mitigated the risk of communication errors and would have facilitated a more coordinated response between hospital and primary care services. Due to the prolonged nature of treatment of TB and requirement for strict adherence to the drug regimen, consideration of the impact of this treatment on quality of life is essential for patient collaboration. The effects of the potential restriction of employment and competing social priorities need to be preempted and addressed in order to optimise treatment adherence. Early involvement of Indigenous health workers and the identification of a case manager is recommended to support individualised and culturally specific, patient-centred care.

\section{REFERENCES:}

1 Tiemersma EW, van der Werf MJ, Borgdorff MW, Williams BG, Nagelkerke NJ. Natural history of tuberculosis: duration and fatality of untreated pulmonary tuberculosis in HIV negative patients: a systematic review. PloS One 2011; 6(4): e17601. https://doi.org/10.1371/journal.pone.0017601 PMid:21483732

2 World Health Organization. Global tuberculosis report 2017. Geneva: World Health Organization, 2017.

3 Williams H, Phelan P. The epidemiology, mortality and morbidity of tuberculosis in Australia: 1850-94. Journal of Paediatrics and Child Health 1995; 31(6): 495-498. https://doi.org/10.1111 /j.1440-1754.1995.tb00870.x PMid:8924298

4 Toms C, Stapledon R, Waring J, Douglas P. Tuberculosis notifications in Australia, 2012 and 2013. Communicable Diseases
Intelligence Quarterly Report 2015; 39(2): E217-E235.

5 Simpson G, Clark P, Knight T. Changing patterns of tuberculosis in Far North Queensland. Medical Journal of Australia 2006; 184(5): 252. https://doi.org/10.5694/j.1326-5377.2006.tb00219.x PMid:16515442

6 Queensland Health. Health Service Directive - Tuberculosis control: protocol for the control of tuberculosis. Protocol QHHSDPTL-040-1:2018. 2018. Available: https://www.health.qld.gov.au/_data/assets/pdf_file/0024/155175 /qh-hsdptl-040-1.pdf (Accessed 14 December 2018).

7 Nahid P, Dorman SE, Alipanah N, Barry PM, Brozek JL, Cattamanchi A, et al. Official American Thoracic Society/Centers for Disease Control and Prevention/Infectious Diseases Society of 
America Clinical Practice Guidelines: Treatment of drug-susceptible tuberculosis. Clinical Infectious Diseases 2016; 63(7): e147-e195. https://doi.org/10.1093/cid/ciw376 PMid:27516382

8 World Health Organization, Stop TB Initiative. Treatment of tuberculosis: guidelines. 4th Edn. 2010. Available: https://apps.who.int/iris/bitstream/handle/10665/44165 19789241547833_eng.pdf (Accessed 15 May 2019).

9 Munro SA, Lewin SA, Smith HJ, Engel ME, Fretheim A, Volmink J. Patient adherence to tuberculosis treatment: a systematic review of qualitative research. PLoS Medicine 2007; 4(7): e238.

https://doi.org/10.1371/journal.pmed.0040238 PMid:17676945

10 Bojovic O, Medenica M, Zivkovic D, Rakocevic B, Trajkovic G, Kisic-Tepavcevic D, et al. Factors associated with patient and health system delays in diagnosis and treatment of tuberculosis in Montenegro, 2015-2016. PloS One 2018; 13(3): e0193997. https://doi.org/10.1371/journal.pone.0193997 PMid:29522545

11 Sreeramareddy CT, Panduru KV, Menten J, Van den Ende J. Time delays in diagnosis of pulmonary tuberculosis: a systematic review of literature. BMC Infectious Diseases 2009; 9(1): 91. https://doi.org /10.1186/1471-2334-9-91 PMid:19519917

12 Getnet F, Demissie M, Assefa N, Mengistie B, Worku A. Delay in diagnosis of pulmonary tuberculosis in low- and middle-income settings: systematic review and meta-analysis. BMC Pulmonary Medicine 2017; 17(1): 202. https://doi.org/10.1186 /s12890-017-0551-y PMid:29237451

13 Ward J, Siskind V, Konstantinos A. Patient and health care system delays in Queensland tuberculosis patients, 1985-1998. International Journal of Tuberculosis and Lung Disease 2001; 5(11): 1021-1027.
14 Lawrence M, Dodd Z, Mohor S, Dunn S, De Crespigny C, Power $C$, et al. Improving the patient journey: achieving positive outcomes for remote Aboriginal cardiac patients. 2009. Available: https://www.lowitja.org.au/content/Document/Lowitja-Publishing /Improving_the_patient_journey.pdf (Accessed 3 May 2019).

15 Suwankeeree W, Picheansathian W. Strategies to promote adherence to treatment by pulmonary tuberculosis patients: a systematic review. International Journal of Evidence-Based

Healthcare 2014; 12(1): 3-16. https://doi.org/10.1097

/01.XEB.0000444614.17658.46 PMid:24685895

16 Fraser HS, Habib A, Goodrich M, Thomas D, Blaya JA, Fils-Aime $J R$, et al. E-health systems for management of MDR-TB in resourcepoor environments: a decade of experience and recommendations for future work. Medlinfo 2013; 627-631.

17 Langloh de Dassel J, Ralph AP, Cass A. A systematic review of adherence in Indigenous Australians: an opportunity to improve chronic condition management. BMC Health Services Research 2017; 17(1): 845. https://doi.org/10.1186/s12913-017-2794-y PMid:29282117

18 Courtwright A, Turner AN. Tuberculosis and stigmatization: pathways and interventions. Public Health Reports 2010;

125(4_suppl): 34-42. https://doi.org/10.1177 /00333549101250S407 PMid:20626191

19 Kastien-Hilka T, Rosenkranz B, Sinanovic E, Bennett B, Schwenkglenks M. Health-related quality of life in South African patients with pulmonary tuberculosis. PloS One 2017; 12(4): e0174605. https://doi.org/10.1371/journal.pone.0174605 PMid:28426759

This PDF has been produced for your convenience. Always refer to the live site https://www.rrh.org.au/journal/article/5552 for the Version of Record. 